\title{
Adaptive Instrumentation Framework Based on Genetic Algorithm
}

\author{
Zhou Lijuan ${ }^{1}$, Li Xiaoyu ${ }^{1}$, Hu Jianya ${ }^{1}$ and Zhang Shudong ${ }^{1+}$ \\ ${ }^{1}$ Information Engineering College, Capital Normal University, Beijing 100048, China
}

\begin{abstract}
Distributed system monitoring suffer from a serious waste of resources when monitoring target programs to obtain information about program operations. In this paper, a framework for adaptive selection of insertion probe positions based on Genetic Algorithm is designed. The monitoring needs of the data are evaluated by execution time and whether exceptions are reported, and the location of the insertion probes is selected according to the evaluation results. The experimental results show that the method proposed in this paper is superior to Pinpoint in terms of performance and causes less interference to the target program.
\end{abstract}

Keywords: distributed system monitoring, genetic algorithms, adaptive instrumentation.

\section{Introduction}

As the foundation of information technology such as cloud services, grid computing, and highperformance computing, distributed systems are becoming increasingly important in modern information technology. Currently, more and more systems are adopting distributed architecture to achieve rational and efficient use of system resources and enhance system scalability. With the popularity and widespread application of distributed systems, distributed system monitoring technology [1] has gradually developed and received the attention of many researchers. Dapper [2] is a large-scale distributed cluster tracking system developed by Google. Based on the thesis design of Google Dapper, Twitter has in turn developed Zipkin [3]. In China, many companies have developed their own distributed tracking systems, such as Taobao's Hawkeye, Sina's Watchman, Jingdong's Hydra, etc.

At present, distributed system tracking tools also have the disadvantage of high resource consumption due to extensive instrumentation and high perturbation of the target program. The root cause of this is the large amount of procedural information generated and collected during the monitoring process. Therefore, adaptive regulation of monitoring content can effectively control the consumption of extensive instrumentation, reduce the amount of data generated and avoid unnecessary waste. The current research on adaptive instrumentation includes: $\mathrm{Yu}$ et al. [4] proposed an adaptive instrumentation framework for program abnormality diagnosis for the .net framework. However, large-scale distributed systems have numerous and complex threads that are difficult to collect and perform comprehensive analysis. Jeswani et al. [5] performed weight analysis for each instrumentation point, and adaptively decided whether to insert a probe by weight analysis. The disadvantage is that a large amount of data needs to be collected for weight analysis, resulting in waste of resources. Karianakis et al. [6] used performance data to predict the response time, and judged whether there was an abnormality by response time, and then adaptively selected the node with abnormality for instrumentation. However, in order to obtain various performance data, collecting response time from multiple dimensions in a fine-grained manner also generates a large amount of resource consumption.

The adaptive instrumentation framework based on Genetic Algorithm proposed in this paper can be realized in two main steps: (1) to select suitable classifiers for classification by selecting reasonable features

\footnotetext{
Corresponding author. Tel.: 68901370; fax:68416830.

E-mail address: zsd@cnu.edu.cn.
} 
of user-defined functions and basic class library functions and third-party insertion library functions; (2) to evolve the optimal combination by genetic manipulation of insertion probe combinations of user-defined functions by genetic algorithm.

\section{Adaptive Instrumentation Framework}

\subsection{Data Collection}

In this paper, a function-level probes are used, so the function information of the program is collected as data for classification. This article collects information on commonly used functions in Java class libraries, including user-defined functions, functions from third-party plug-in libraries and functions in the Java basic class libraries. Finally, a total of 76873 function information was collected including functions defined by basic classes in seven Java public packages including java.lang, java.util, java.sql, and three common Java third-party libraries (Commons Math, Commons Lang, Commons IO).

\subsection{Classifier Based on Naïve Bayesian Algorithm}

The Naïve Bayesian classifier [7] is a commonly used classification model based on Bayes' theorem, which treats all properties as independent based on the "attribute condition independence assumption [8]". The advantages are simple algorithms and low resource consumption.

In order to distinguish functions from different sources, the following characteristics were selected as criteria for classification: A function is generally called by its function name and arguments. Usually userdefined functions follow a certain naming convention by setting prefixes or namespaces. Function overloading causes some functions to have the same function name, but different numbers or types of arguments. Therefore, the function name and arguments are chosen as the first feature. Function prototypes of Java Basic Class Library are stored in the <JAVA_HOME > directory; prototypes of functions provided by third-party plug-in libraries are generally included in a separate Jar package; and user-defined functions are stored in the corresponding directory of the application; therefore, the paths of different functions are used as a second feature. Functions in the basic library do not need to be defined by the user, nor do they need to declare the type before use, so that they can be called directly in the program; functions in the third-party plug-in library are also defined in advance in the third-party library, and the user can access and operate through the provided interface; user-defined functions require the user to define the function itself, and to declare the type of the called function before calling. Therefore, whether the function is declared before the call can be used as a third feature. The generation or modification of a project changes the content of the project file and the final modification time of the file. Checking that the final modification time of the function is consistent with the program can be used as a fourth feature to identify user-defined functions.

\subsection{Adaptive Instrumentation Based on Genetic Algorithm}

Genetic Algorithm [9] is a population-based heuristic algorithm that uses three genetic operations, selection, crossover, and variation, to simulate natural selection and natural genetic laws in the biological world, using iteration to select individuals with good adaptability and eliminate poorly adapted individuals. The genetic algorithm is chosen to solve the optimal probe combination in this paper mainly because it uses binary coding, which is more suitable for solving the 0-1 nonlinear optimization problem than other parallel optimization algorithms, and treats the sequence of function calls as chromosomes and each function in the sequence as a gene, which is more consistent with the representation of the genetic algorithm.

The screened user-defined functions are encoded as binary genes, with each gene bit corresponding to a custom function, e.g. a chromosome encoded as:0111101100. Where "0" means do not insert a probe to the function, "1" means insert a probe. The digits of a binary number represent the number of custom functions.

Different functions are called according to different requests from the system and different combinations of insertion probes under the same call to form the initial population. $c_{i}$ denotes chromosome $i(i=1,2, \ldots, n)$ and $\mathrm{n}$ represents the size of the initial population.

$$
\mathrm{C}=\left(\mathrm{c}_{1}, \mathrm{C}_{2}, \ldots, \mathrm{c}_{\mathrm{n}}\right)
$$

The fitness function chosen in this paper is related to the monitoring needs of the system, the execution time is an important indicator of the presence of anomalies in the system, the presence of delayed or time-out 
anomaly data is more valuable than normal time data, it can help maintenance and developers detect and diagnose system anomalies. In addition, if the system already reports an exception, the program reporting the exception must be staked to obtain more execution information to diagnose the cause of the exception, so whether to report an exception is also an important criterion to judge whether the data has higher monitoring needs. Ultimately, this paper chooses two criteria for defining the fit function: execution time and whether to report an exception, which is expressed as:

$$
\mathrm{y}=\sum_{i=1}^{m} \omega_{\mathrm{i}} \mathrm{t}_{i}+\sum_{i=1}^{m} \lambda_{i}
$$

$\omega_{\mathrm{i}}$ is a weighting factor of execution time and can be calculated by the following formula:

$$
\omega=\left\{\begin{array}{l}
\frac{\mathrm{t}}{\mu}-1, \mathrm{t}<2 \mu \\
1, \mathrm{t} \geq 2 \mu
\end{array}\right.
$$

and,

$$
\mu=\frac{1}{n} \sum_{i=1}^{\mathrm{k}} t_{i}
$$

$t_{i}$ indicates the execution time of the $i_{t h}$ function, $\mu$ is the average execution time of a function.

The value of $\lambda$ indicates whether the function reports an exception.

$$
\lambda= \begin{cases}0, & \text { normal } \\ 1, & \text { anomalies }\end{cases}
$$

Selection operation: after calculating the value of the chromosomal fitness function, the individuals implementing the cross are identified by the roulette selection method. The selection probability is calculated for each individual based on the fit value, and then a random number is generated evenly between [0,1], and this random number is used as a selection pointer to determine the selected individual.

Crossover operation: The crossover algorithm of double-point crossover is used, $M=<\mathrm{M}_{1}, \mathrm{M}_{2}, \ldots, \mathrm{M}_{\mathrm{m}}>$ and $\mathrm{N}=<\mathrm{N}_{1}, \mathrm{~N}_{2}, \ldots, \mathrm{N}_{\mathrm{m}}>$ are used to represent the two crossover chromosomes, the integers $\mathrm{s}_{1}, \mathrm{~s}_{2}$ are selected from $1 \sim(\mathrm{m}-1)$ as the two crossover points of the double-point crossover method, and the two new chromosomes that perform the crossover operation are represented as:

$$
\begin{aligned}
& <M_{1}, M_{2}, \ldots, M_{s_{1}}, N_{s_{1}+1}, \ldots, N_{s_{2}-1}, M_{s_{2}}, \ldots, M_{m}> \\
& <N_{1}, N_{2}, \ldots, N_{s_{1}}, M_{s_{1}+1}, \ldots, M_{s_{2}-1}, N_{s_{2}}, \ldots, N_{m}>
\end{aligned}
$$

Mutation operation: After encoding using binary method, a position s is randomly selected from 1 to m. If the value on s before mutate is " 0 ", the value on s after mutate is " $1 "$.

Table.1: Classifier training and prediction results

\begin{tabular}{lllll}
\hline category & number of training & number of tags & forecast result & accuracy \\
\hline Third-party library functions & 15115 & 3027 & 5102 & $59.33 \%$ \\
Basic class library function & 33370 & 4896 & 4829 & $98.63 \%$ \\
User-defined function & 13388 & 7077 & 5069 & $71.62 \%$ \\
Total & 61873 & 15000 & 15000 & $86.17 \%$ \\
\hline
\end{tabular}

\section{Experiments and Evaluation}

\subsection{Classifier Experiment Results}

The 61873 data collected were used for training and 15000 for classifier evaluation, and the final experimental results are shown in Tab.1. The functions collected from different sources were classified using 
a Naive Bayesian classifier, and finally 3027 third-party library functions, 4829 Java Basic library functions, and 5069 user-defined functions were correctly classified, with a correct rate of $86.17 \%$. According to the statistical results, there are some user-defined functions misclassified as third-party library functions, the main reason is that these user-defined functions are not stored in the specified path of the target program, and their final modification time does not match the main project.

\subsection{Adaptive Experimental Results}

Data from a simple microservices system were used in this experiment, and a total of 15 custom functions were filtered through the classifier to generate original population data based on different operation requests and different combinations of insertion probes. The main parameters involved in the algorithm are set as follows: cross probability $\mathrm{pc}=0.7$, mutation probability $\mathrm{pm}=0.08$, overall population size 500 , number of evolutions 300. Fig. 1 shows the evolution of the fitness values of the population.

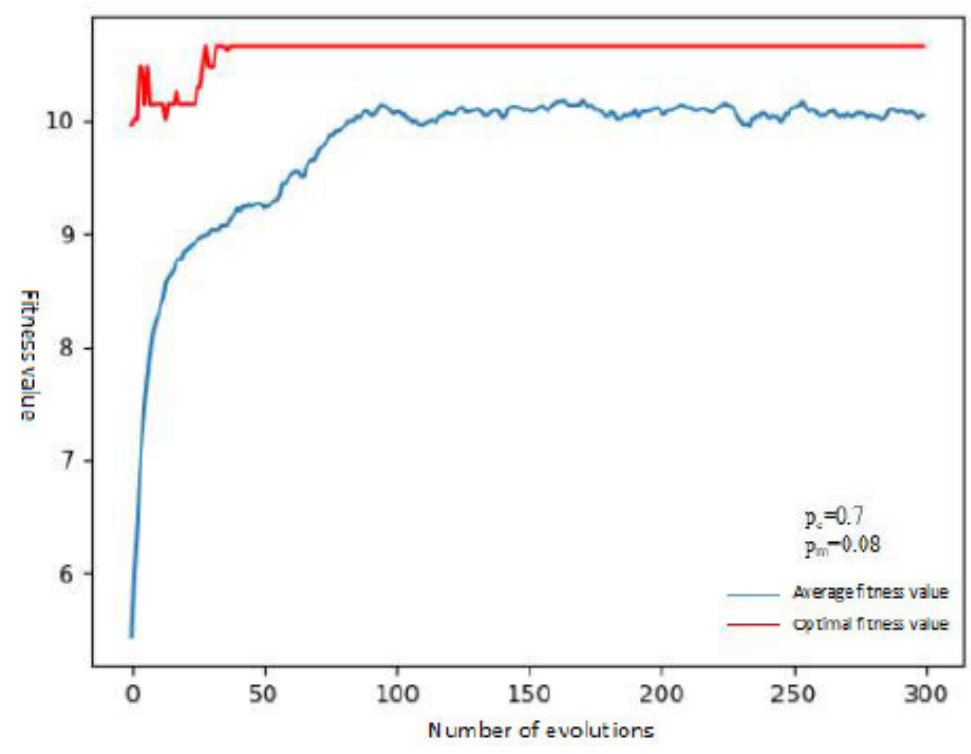

Fig.1: The relationship between fitness values and the number of evolution

The blue line in the figure represents the trend in the mean population fitness values after different evolutionary generations, and the red line represents the change in the best fitness values. From Fig.1, it can be seen that when the evolutionary algebra reaches about 50 times, the maximum value of the degree of adaptation tends to stabilize and its value no longer changes. The main reason for this phenomenon is that a small microservice system was chosen as the experimental subject, which contains only 15 user-defined functions.

\subsection{Experimental results of an adaptive instrumentation}

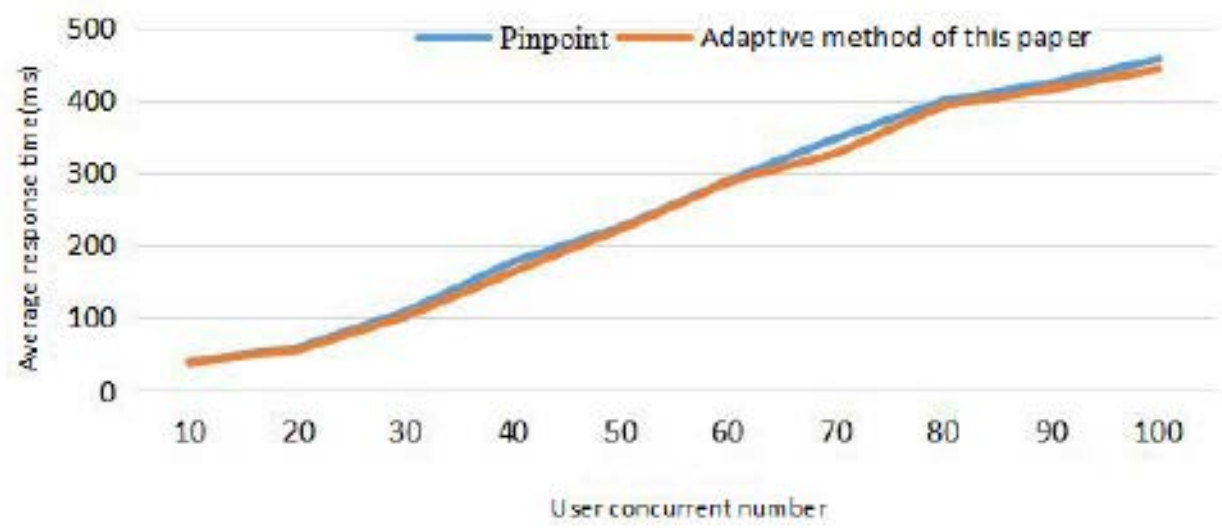

Fig.2: Change in average response time under different user loads 


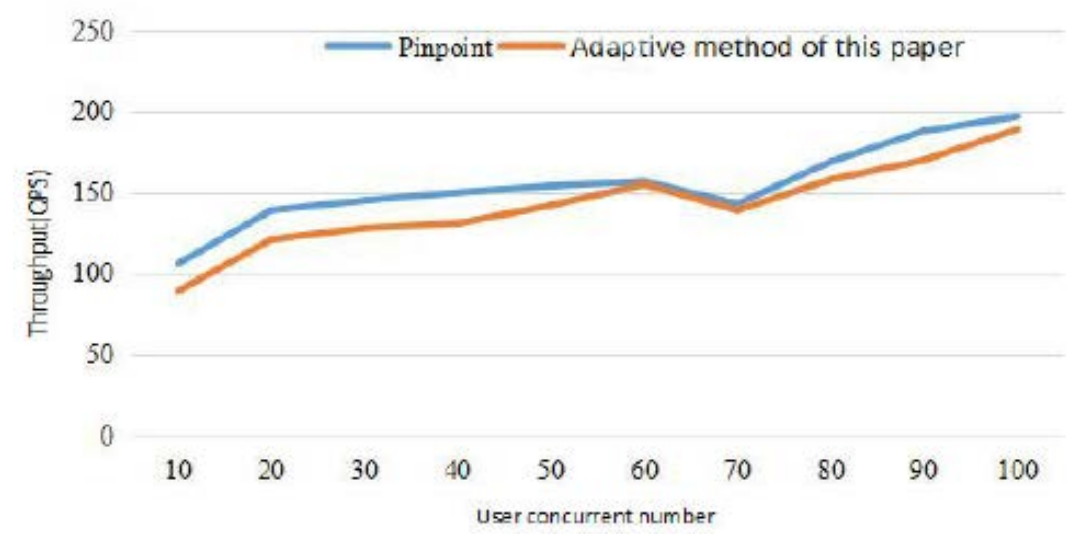

Fig.3 Change in service throughput under different user loads

Experimental selection of the shopping site Heat Clinic [10] written in Java as the target program, which is based on the open source e-commerce website framework Broadleaf, with a multi-layered architecture, mainly using the SpringBoot framework development. During the experiment, the website was deployed to the Tomcat application server, the data was stored using the MySQL database, the load was generated using JMeter, and the performance of the system was observed under different stress loads. The main performance metrics viewed are page response time and throughput [11], which are used to observe the interference of the pile driving tool on the target system. The anomalies during the experiment were simulated by artificially injecting thread delays into the site. The operating system used in the experiment was Windows 10, the processor was Intel Core i7-4790u, and the memory size was 8GB. Fig.2 shows the variation of the average response time applied when different insertion probe strategies are adopted for different numbers of concurrent users. Fig.3 shows the variation in service throughput with different insertion probe strategies for different concurrent user counts.

Based on the experimental results shown in the above two images, it can be seen that by using JMeter to increase the load of access to the Heat Clinic website and simulate the business access of concurrent users, the strategy of adaptive selection of insertion probes based on the genetic algorithm proposed in this paper is better than Pinpoint [12] in terms of performance and causes less interference to the target program.

\section{Conclusion}

In this paper, we improve on the disadvantage of high resource consumption of the traditional insertion probe strategy, and propose an adaptive instrumentation based on Genetic Algorithm, which reduces interferences with the target program compared to Pinpoint. However, there is still room for improvement in this paper: current adaptive instrument will take too long to execute. In addition to this, there are times when programs that do not report exception information have performance issues. It is also too simple to consider execution time as the only factor to measure performance. As a result, better adaptive rules can be chosen to measure the monitoring needs at the inserted probe position and obtain more procedural information to help more accurately locate anomalies in the analysis system.

\section{Acknowledgements}

This research was supported by National Key R\&D Program of China（2018YFB1402900）

\section{References}

[1] Liotta A. Distributed Monitoring: Methods, Means and Technologies. In: Leondes C.T. (eds) Intelligent Knowledge-Based Systems. Springer, Boston, MA, 2005:965-998.

[2] Sigelman B H, Barroso L A, Burrows M, et al. Dapper, a large-scale distributed systems tracing infrastructure[R]. Technical report, Google, Inc, 2010.

[3] Zipkin. http://twitter.github.com.io/zipkin/.

[4] Yu Y, Rodeheffer T, Chen W. RaceTrack: Efficient detection of data race conditions via adaptive tracking[J]. ACM 
SIGOPS Operating Systems Review, 2005, 39(5):221-234.

[5] Jeswani D, Natu M, Ghosh R K. Adaptive monitoring: A framework to adapt passive monitoring using probing[C]// Network \& Service Management. IEEE, 2012:350-356.

[6] Karianakis N. Sampling Algorithms to Handle Nuisances in Large-Scale Recognition[J].2017.

[7] Gong Xiujun. Bayesian Learning Theory and Its Application [D]. Graduate School of Chinese Academy of Sciences (Institute of Computing Technology), 2002.

[8] Zaidi N, Cerquides J, Carman M, et al. Alleviating Naive Bayes attribute independence assumption by attribute weighting[J]. Journal of Machine Learning Research, 2013, 14(1):1947-1988.

[9] Bian Xia, Mi Liang. Research progress in genetic algorithm theory and its application[J]. Journal of Computing Applications, 2010, 27(07): 2425-2429+2434.

[10] Broadleaf online demo. http://demo.broadleafcommerce.org/.

[11] Yuan Xinchen, Li Haibo, Wang Wei, Tang Zhen, Ren Zhongshan, Zheng Yingying. Distributed application automation tracking method based on program analysis[J]. Journal of Computer Systems, 2016,25(11):35-40.

[12] Pinpoint. https://github.com/naver/pinpoint. 